\title{
Disruption of early pregnancy by direct and indirect exposure to novel males in mice: comparison of influences of preputialectomized and intact males
}

\author{
D. deCatanzaro, R. Zacharias and C. Muir \\ Department of Psychology, McMaster University, Hamilton, Ontario, L8S 4K1, Canada
}

\begin{abstract}
Inseminated female CF-1 mice (Mus musculus) were exposed on days 1 to 5 of pregnancy to unfamiliar outbred males. In the first experiment, inseminated females were each housed directly with the sire, a preputialectomized male, or an intact male. Both types of novel male attempted to mate with the female during this period, unlike the sire. Reinsemination occurred in a significant proportion of the females that were exposed to novel males; this effect was equivalent for preputialectomized and intact males. In two subsequent experiments, we refined a paradigm of indirect exposure to novel males through a wire-mesh grid, which prevents mating and reinsemination. Two or three males housed directly above each female through a grid disrupt pregnancy in most cases, but housing the males below the females is much less likely to do so. In a final experiment, each inseminated female was housed below two males that were either preputialectomized or sham-preputialectomized. Whereas 29 of 33 undisturbed controls were parturient, only eight of 32 females exposed to sham-preputialectomized males and six of 32 exposed to preputialectomized males were parturient. These results suggest that nonvolatile pheromones are involved in novel-maleinduced pregnancy disruptions, but that preputial gland emissions are not necessary for such disruptions.
\end{abstract}

\section{Introduction}

Environmental changes can have adverse effects upon early pregnancy in mammals (deCatanzaro and MacNiven, 1992). Gestation can be disrupted before or during the period of intrauterine implantation of fertilized ova by diverse stressors, such as chronic restraint (Wiebold et al., 1986; deCatanzaro et al., 1994), daily handling (Runner, 1959), loud noise (Zondek and Tamari, 1967), high ambient temperatures (Hsu, 1948), environmental and social changes (Bronson et al., 1964), and exposure to predators (deCatanzaro, 1988). Exposure to novel males can also disrupt early pregnancy in various rodent species (Bruce, 1959, 1960a, b, 1963; deCatanzaro and Storey, 1989; deCatanzaro et al., 1995a, b), a phenomenon known as the 'Bruce effect'.

Several reports suggest a pheromonal mediation of the Bruce effect. Parkes and Bruce (1962) reported that exposing inseminated females to cages soiled by males was sufficient for pregnancy disruption, but only when cages were extremely soiled and air exchange was limited. The Bruce effect was prevented when the olfactory bulbs (Bruce and Parrott, 1960) or the vomeronasal organs (Bellinger et al., 1980; Lloyd-Thomas and Keverne, 1982) of the females were lesioned or the lateral olfactory tract was transected (Rajendren and Dominic, 1986), although one study found that the original sire could disrupt pregnancy when the vomeronasal system of

Received 9 August 1995 the female was damaged (Keverne and de la Riva, 1982). There are also reports (Dominic, 1965, 1966a, b) of strong pregnancy disruptions when male urine was applied on the nasal area of normal females, and that urinary proteins salted out of male urine similarly disrupt pregnancy (Marchlewska-Koj, 1977, 1981). However, concerns have been raised (deCatanzaro and MacNiven, 1992) about human handling and genital stimulation in these latter studies, and indirect inferences about pregnancy outcome based on vaginal smears rather than litter production. Efforts to disrupt pregnancy by male urine alone, either placed in the female's bedding or topically painted on its nasal region, have not been successful in this laboratory (deCatanzaro et al., 1995b). We have found that the Bruce effect depends upon androgen concentrations of novel males; it is eliminated by castration and reinstated by testosterone administration, and no effect occurs unless inseminated females are in close proximity to novel males (deCatanzaro and Storey, 1989; deCatanzaro et al., 1995a, b).

Male pheromones are implicated in stimulation of ovulation (Whitten, 1956) and acceleration of puberty (Vandenbergh, 1967) in female mice. These chemosignals are present in male urine and their production is under androgen control (Bronson and Whitten, 1968; Vandenbergh, 1969). Inseminated female mice avoid the odour of novel males (Drickamer, 1989). However, very little is known about the chemical structure and the site of synthesis of male pheromones. The preputials, sebaceous glands situated between the skin and body wall anterior to the external genitalia, seem to be likely candidates 
as sources of pheromones. They are prominent in male mice but normally vestigial in females (Mugford and Nowell, 1971; Raso et al., 1992). The absolute mass of the preputial glands relates inversely to population density in male mice (Christian, 1955). The degree of male preputial gland activity is apparantly related to social experience, especially aggression (Hucklebridge et al., 1972). Evidence suggests that these glands affect fighting behaviour through the release of an aggressionpromoting pheromone (McKinney and Christian, 1970; Mugford and Nowell, 1970; Jones and Nowell, 1973). Testosterone administration induces a hypertrophy of the female's preputial glands while making their urine more aggressionpromoting or less aggression-inhibiting (Mugford and Nowell, 1971). Preputial gland secretions are attractive to animals of the opposite sex in rats (Noble and Collip, 1941; Orsulak and Gawienowski, 1972; Gawienowski et al., 1975; Thody and Dijkstra, 1978) and mice (Bronson and Caroom, 1971). They may also regulate anogenital licking (Brouette-Lahlou et al., 1991) and aid in maternal discrimination of the sex of pups (Moore and Samonte, 1986). One report (Marchlewska-Koj et al., 1990) indicates that male preputial glands as well as salivary glands are sources of pheromones that stimulate oestrus in female mice.

The present study was designed to examine whether male preputial glands are responsible for the Bruce effect. Inseminated female mice were exposed to novel males, either preputialectomized or not, during the first 5 days after insemination. It was assumed that, insofar as preputial pheromones mediate the Bruce effect, preputialectomized males would fail to disrupt pregnancy. This was examined through a direct exposure paradigm, in which females can be reinseminated by novel males, and an indirect exposure paradigm, in which males cannot mate with females because of separation by wire-mesh. Inseminated females were allowed to bear litters to provide a direct measure of pregnancy outcome, and human handling was minimized during the procedures.

\section{Materials and Methods}

\section{Animals and insemination procedures}

The experimental subjects and inseminating males were CF-1 strain mice bred in this laboratory from stock obtained from Charles River Breeding Farms (La Prairie, Quebec). The novel males were HS (heterogeneous strain), bred in this laboratory from stock originally obtained from the Department of Zoology (University of Toronto). The animal colony room was maintained at $21^{\circ} \mathrm{C}$ under a reversed $14 \mathrm{~h}$ light: $10 \mathrm{~h}$ dark cycle. Housing, rearing and maintenance were as described by deCatanzaro et al. (1995b). At 65-100 days of age, nulliparous females were placed individually in a cage containing a male at the onset of the dark phase of the light cycle. Females were inspected for sperm plugs as described by deCatanzaro et al. (1995b); those with plugs were designated as subjects and the day of detection of the plug was designated as day 0 of pregnancy. On day I, I-3 h after the start of the dark phase of the light cycle, females were assigned randomly to one of the experimental conditions, with date of insemination counterbalanced across conditions.

\section{Preputialectomy}

The preputial glands of male mice consist of a pair of leaf-shaped ectodermal exocrine glands opening to the exterior on either side of the urethral meatus (Fekete, 1941; Bronson and Caroom, 1971). They are located bilaterally between the skin and the body wall anterior to the external genitalia. They are yellowish-brown, dorsoventrally flattened, measuring approximately $7 \mathrm{~mm} \times 5 \mathrm{~mm}$, although they are quite variable in size among individuals. For removal of the glands, the animals were anaesthetized with sodium pentobarbitol. Two incisions, each measuring approximately $5 \mathrm{~mm}$, were made on either side of the external genitalia, or, if possible, both glands were removed via a single incision. The glands were found immediately under the skin layer. Once located, they were snipped off at the stem. In some individuals, a thin membranous tissue was present either over or between the two glands; this was snipped as necessary. Once the glands were removed, the skin incision was closed by a wound clip and animals were kept warm on a heating pad until normal activity returned. The wound clip was removed without anaesthesia 1 week after surgery. For sham-preputialectomized animals, the procedures of anaesthesia, skin incisions, and recovery were the same as in those receiving the full surgery, but the preputial glands were left undisturbed. All surgically treated males were allowed at least 2 weeks for recovery before they were used as stimulus animals in the experiments. Subsequent to the experiments, a subset of preputialectomized males was killed for histology at the site of surgery to confirm that the glands had not regenerated.

\section{Direct exposure paradigm}

In an initial experiment, each inseminated female was assigned randomly on day 1 of pregnancy to housing in a clean cage with one of the following animals: (1) a novel intact HS male, (2) a novel preputialectomized HS male, or (3) the sire of the pregnancy (control condition). Each female remained housed directly with the stimulus male for $120 \mathrm{~h}$. Behavioural interactions between the male and the inseminated female were monitored during the first day of such housing. Immediately after the experimental procedures began, each male-female pair was observed continuously for $2 \mathrm{~h}$. During this observation period, a trained observer recorded the occurrence of sexual activity (including mounts and intromissions; see McGill, 1965; deCatanzaro and Gorzalka, 1979). Additional observation periods of 10 min of the same behaviour were taken on each pair at about $5 \mathrm{~h}$ and $8 \mathrm{~h}$ after commencement of the dark phase of the normal lighting cycle on that day.

\section{Indirect exposure paradigm}

On day 1 of pregnancy, each inseminated female was either designated as a no-male-exposure control subject, or began $120 \mathrm{~h}$ of indirect exposure to one or more novel males, which was accomplished in a double-decker apparatus separating the female from the males by a wire-mesh partition, as described by deCatanzaro et al. (1995b). Initial parametric work was undertaken to establish a strong baseline effect using various numbers of intact novel HS males. The two compartments of 
the double-decker apparatus were separated by a wire-mesh grid with openings measuring $1 \mathrm{~cm} \times 1 \mathrm{~cm}$. In one experiment, each female was housed in the lower compartment of such an apparatus, exposed to one, two or three HS males in the upper compartment. In a subsequent experiment, each female was housed in the upper or the lower compartment so that she was above or below three novel HS males in the other compartment.

Subsequently, as it was established that a strong and reliable pregnancy disruption occurred when each inseminated female was housed below multiple males, a major experiment was begun with females assigned randomly to three conditions: (1) housed below no male (control condition), (2) housed below two novel preputialectomized HS males, and (3) housed below two novel sham-operated HS males. Incidental aggression between the paired novel males was prevented by placing a partition between them in each upper compartment of the experimental apparatus. This was achieved initially via a wiremesh grid that divided the upper compartment in two. For subsequent replications, the apparatus was modified such that a double Plexiglas partition in the upper compartment prevented all contact between the two males, and the wire mesh between the upper and lower compartments was replaced with finer mesh (openings measuring about $0.5 \mathrm{~cm} \times 0.5 \mathrm{~cm}$ ).

\section{Pregnancy outcome measures}

At the end of the $120 \mathrm{~h}$ period of exposure to novel males, each female was housed individually in a clean cage with fresh bedding and was left undisturbed for the duration of gestation. On day 18 after the detection of the initial sperm plug, females were checked on three occasions each day for parturition. Such inspections continued until 30 days after detection of the plug. Pregnancy outcome was measured by counts of the number of pups born, duration of gestation, and number of pups 3 days after birth (cannibalizations and stillbirths were recorded). In the direct exposure paradigm, coat colour was measured by retaining pups until they were 10 days of age; this was taken as an indication of sire, since CF-1 (original sire) litters are homogeneously white whereas HS (novel sire) litters are multicoloured.

\section{Results}

\section{Direct exposure paradigm}

All novel males, except one intact and one preputialectomized male, showed some mounting behaviour towards the previously inseminated females during the interval samples, whereas only three of the original sires did so. The mean number of mounts ( \pm SEM) was $0.4 \pm 0.3$ for sires, $35.2 \pm 4.4$ for preputialectomized males, and $50.8 \pm 6.2$ for intact males. Generally, females resisted such mounting, but intromission by seven intact novel males, seven preputialectomized novel males, and no sires was observed. The mean number of intromissions was $1.7 \pm 0.8$ for preputialectomized males and $3.5 \pm 1.9$ for intact males. No aggressive interactions between males and females were observed. Analysis of variance among the three experimental conditions was significant for number of

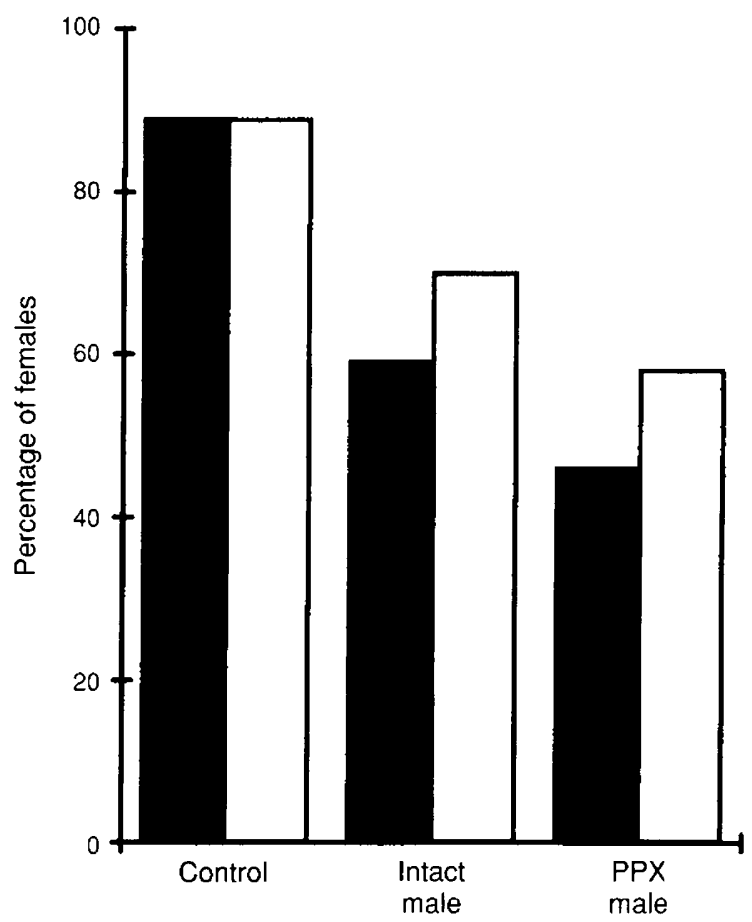

Fig. 1. Percentage of females with litters attributable to the original sire after direct exposure to the sires (control), novel preputialectomized (PPX) outbred males, or novel intact outbred males on days 1-5 of pregnancy, by criteria of coat colour and duration of gestation (ם) or coat colour alone $(\square)$.

mounts, $F(2,79)=35.24, P<0.0001$, but not for number of intromissions, $F(2,79)=2.12, P=0.1253$. Multiple comparisons (Newman-Keuls' test, $P<0.05$ ) showed differences for number of mounts among all three conditions. Although most females were parturient in each condition, the duration of gestation and the coat colours suggested that the original pregnancy was replaced in a subset of females exposed to intact or preputialectomized males. In the sire-exposed (control) condition, 24 of 27 females were parturient; all litters yielded exclusively white pups, and the maximum duration of gestation was 21 days. Of 27 females exposed to intact males, two were not parturient and the duration of gestation of nine of the litters produced was of 23 days or greater, or the litter contained coloured pups. Of 26 females exposed to preputialectomized males, four were not parturient and the duration of gestation of nine of the litters was 23 days or greater, or contained coloured pups. Given that we have never observed a gestation exceeding 22 days for CF-1 mice by the original sire in any study in this laboratory, and CF-I mice are homogeneously albino while HS mice are multicoloured, it was inferred that the pregnancies were replaced by the novel male in such cases. An albino litter is possible but not common with $\mathrm{HS} \times \mathrm{CF}-1$ crosses. Figure 1 illustrates the number of females in each group bearing litters that were attributable to the original sire, using these criteria as well as a more conservative estimate that ascribed a pregnancy disruption only to cases in which a litter was absent or coloured, excluding consideration of duration of gestation. A test of association comparing conditions was significant with both the stronger set of criteria $\left(\chi^{2}(2)=11.22, P<0.005\right)$ and the more conservative set of criteria $\left(\chi^{2}(2)=7.06, P<0.05\right)$. 


\section{Indirect exposure paradigm}

Initial parametric work indicated that loss of pregnancy was more likely to occur in females with greater numbers of males in their vicinity, and that such disruption was more probable when males were housed above females than when they were housed below females. Intense intermale aggression was observed in a subset of grouped males housed near females. In the experiment in which the number of males above females was varied, 16 of 19,8 of 15,4 of 15 , and 2 of 15 females were parturient, respectively, with zero, one, two, or three novel males. A test of association between conditions and the presence or absence of parturition showed significance $\left(\chi^{2}(3)=20.12, P<0.001\right)$. The number of pups born, with zeros assigned to nonparturient females, was, respectively, $10.0 \pm 1.2,6.9 \pm 1.8,3.1 \pm 1.4$, and $1.8 \pm 1.3$; analysis of variance was significant $(F(3,60)=7.43, P<0.0005)$ and multiple comparisons identified difference between conditions with zero versus two or three males, and with one versus three males. In the experiment in which three HS males were housed above or below each female, 14 of 16 control females, 3 of 16 females housed below males, and 10 of 16 housed above males were parturient $\left(\chi^{2}(2)=15.75, P<0.001\right)$. The number of pups born was, respectively, $10.5 \pm 1.1,2.2 \pm 1.2$, and $7.2 \pm 1.5$; analysis of variance was significant $(F(2,45)=10.50$, $P<0.0005)$ and multiple comparisons identified a difference between the condition with males above and the two other conditions.

Figure 2 gives data for the proportion parturient in each condition of the experiment comparing the influences of two preputialectomized males and two sham-preputialectomized males. Most females (29 of 33) in the control group produced litters, but substantially fewer did so in either the preputialectomized novel male or the sham-preputialectomized novel male conditions. The effect was equally present when males were separated by a wire grid ( 2 of 11 females exposed to preputialectomized males and 1 of 11 sham-exposed females were parturient) as when males were separated by a Plexiglas barrier (4 of 21 females exposed to preputialectomized males and 7 of 21 sham-exposed females were parturient) so data were combined. A test of association between conditions and the presence or absence of parturition showed significance $\left(\chi^{2}(2)=38.69, P<0.001\right)$. A test of association comparing just the control and preputialectomized groups was similarly significant $\left(\chi^{2}(I)=31.23, P<0.001\right)$ as was one comparing the control and the sham-operated groups $\left(\chi^{2}(1)=26.20\right.$, $P<0.001$ ) but not comparing the preputialectomized and sham-operated groups. The number of pups born to control females was $10.36 \pm 0.74$; for females exposed to preputialectomized males, $1.97 \pm 0.74$; and, for those exposed to sham males, $3.34 \pm 1.05$. Analysis of variance on this measure was significant $(F(2,94)=27.83, P<0.0001)$ and multiple comparisons identified a difference between the control condition and each of the other two conditions, but not between the preputialectomized and sham conditions.

\section{Histology}

Ten animals that had been preputialectomized were killed, four at 2 months after the surgery and six others at 5 months

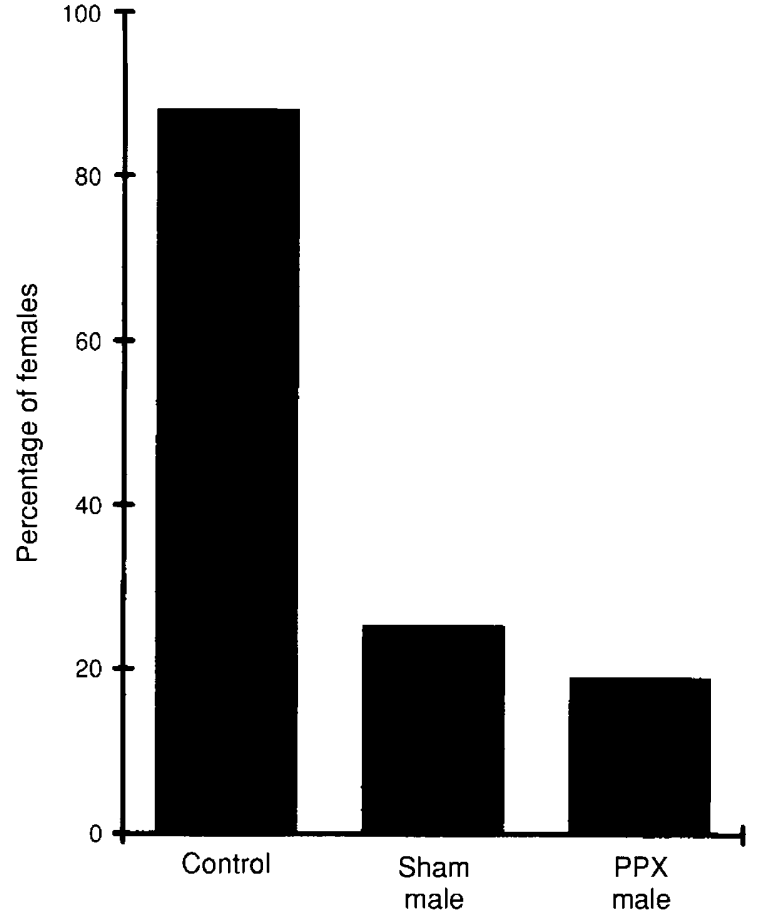

Fig. 2. Percentage of females delivering litters after housing during days 1-5 of pregnancy below two novel outbred males that were either preputialectomized (PPX) or sham-preputialectomized (Sham).

after the surgery. In each case, the former site of the preputial glands could be identified. In all animals, full removal of the preputial glands was confirmed, and in no case was there any sign of regeneration of these glands.

\section{Discussion}

The experiments reported here demonstrate that a Bruce effect can be obtained with both direct and indirect exposure to novel outbred males, but that the effect is qualitatively different in the two cases because of behavioural interactions and reinsemination in the case of direct exposure. Effects are all-or-none and probabilistic, given that some control females do not produce litters and some females exposed to novel males do produce litters, while litter size of parturient females is in the normal range regardless of condition. With either direct or indirect exposure, preputialectomy does not diminish the capacity of novel males to disrupt pregnancy.

With direct exposure of each inseminated female to one novel male, pregnancy disruption is complex, involving various qualities depending upon the individual. The novel male typically attempts to mate with the female; this attempt is met initially with resistance, but some females subsequently permit intromissions. A substantial proportion of females in this strain of mice retain the original pregnancy, whereas others lose the initial pregnancy and often are reinseminated by the novel male, as evidenced by the duration of gestation and coat colours of the litters. The reinsemination rate with CF-1 females in the present study was greater than that found with C57BL females in similar conditions (deCatanzaro and Storey, 1989). 
Since preputialectomized males in the present study replaced at least as many pregnancies as did intact males, we conclude that the preputial gland is not necessary for the Bruce effect under conditions of direct exposure.

The current experiments also refine the paradigm for production of the Bruce effect by indirect exposure. CF-1 females show a higher baseline (control) pregnancy rate than do C57BL females studied elsewhere (deCatanzaro et al., 1995b). Greater numbers of males in their proximity provide a larger effect. These refinements produce a strong and reliable pregnancy disruption, permitting statistical sensitivity to other parametric manipulations. Although intense aggression among males can be a by-product in some groups, apparently stimulated by the proximity of the female, the elimination of this aggression by barriers between males does not diminish the pregnancy disruption. It is noteworthy that exposure to three males disrupts most pregnancies when males are above but not below females. This finding suggests that a nonvolatile chemosignal is involved, given that females housed below males receive far greater quantities of the males' excretions, while there is no obvious behavioural difference between animals in the two conditions.

Marked disruptions of pregnancy occurred when inseminated females were housed below two males that were separated from one another. This was equally the case when the males were preputialectomized as when they were shampreputialectomized. This is perhaps the clearest demonstration of a Bruce effect, uncomplicated by reinsemination and behavioural interactions among animals, and of the irrelevance of the preputial glands. Several human observers in this laboratory have noted independently that a subjectively foul and distinct odour is present in the experimental room when inseminated females are in the double-decker apparatus with males, and that the walls of the males' compartments are smeared with an oily emission during this exposure. There is no apparent difference in the magnitude of these observations with preputialectomized versus intact males. Accordingly, we suggest that a nonvolatile emission of the males is involved in the Bruce effect induced by indirect exposure, that the frequency of pregnancy disruption is a function of the quantity of this emission, but that this emission is not of preputial origin. Other male glands could be the source of such pheromones; some evidence suggests that there are pheromonal emissions from the coagulating glands (Jones and Nowell, 1973), and other sources could include the bulbourethral glands, kidneys and even salivary glands.

The involvement of androgens and oestrogens in both the capacity of males to produce the Bruce effect (Dominic, 1965; deCatanzaro et al., 1995a, b) and endogenously in females, in which intrauterine implantation is very sensitive to increased androgen and oestrogen concentrations is of interest (Harper, 1967; deCatanzaro et al., 1991). Evidence suggests that stress-induced early pregnancy disruptions may be mediated by adrenal androgens and oestrogens (deCatanzaro and MacNiven, 1992; deCatanzaro et al., 1994). Thus, in the context of the Bruce effect, excretions of oestrogens by novel males may merit attention. In contrast to this notion is evidence that the Bruce effect might be subserved in the female by pheromone-altered prolactin concentrations (Bellringer et al., 1980; Rosser et al., 1989). It is clear from an evolutionary- ecological perspective that both chronic stress and strange males are associated with poor prospects for gestation and nurture of young; whether the adaptations to these contingencies are common or distinct remains to be determined.

This research was supported by a grant from the Natural Sciences and Engineering Research Council of Canada to D. deCatanzaro. The authors would like to thank M. Hansen and F. Ali for their careful assistance.

\section{References}

Bellringer JF, Pratt HMP and Keverne EB (1980) Involvement of the vomeronasal organ and prolactin in pheromonal induction of delayed implantation in mice Journal of Reproduction and Fertility 59 223-228

Bronson FH and Caroom D (1971) Preputial gland of the male mouse: attractant function Journal of Reproduction and Fertility 25 279-282

Bronson FH and Whitten WK (1968) Oestrus-stimulating pheromones of mice: assay, androgen dependency and presence in bladder urine Journal of Reproduction and Fertility 15 131-134

Bronson FH, Eleftheriou BE and Garick EI (1964) Effects of intra- and interspecific social stimulation on implantation in deer mice Journal of Reproduction and Fertility 8 23-27

Brouette-Lahlou I, Amouroux R, Chastrette F, Cosnier J, Stoffelsma J and Vernet-Maury E (1991) Dodecyl propionate, attractant from rat pup preputial gland: characterization and identification Journal of Chemical Ecology 17 1343-1354

Bruce HM (1959) An exteroceptive block to pregnancy in the mouse Nature 184105

Bruce HM (1960a) A block to pregnancy in the mouse caused by the proximity of strange males Journal of Reproduction and Fertility 1 96-103

Bruce HM (1960b) Further observations on the pregnancy block in mice caused by the proximity of strange males Journal of Reproduction and Fertility 1 311-312

Bruce HM (1963) Olfactory block to pregnancy among grouped mice Journal of Reproduction and Fertility 6 451-460

Bruce HM and Parrot DMV (1960) Role of olfactory sense in pregnancy block by strange males Science 1311526

Christian JJ (1955) Effect of population size on the weights of the reproductive organs of white mice American Journal of Physiology 181 477-480

deCatanzaro D (1988) Effect of predator exposure upon early pregnancy in mice Physiology and Behavior 43 691-696

deCatanzaro D and Gorzalka BB (1979) Isolation-induced facilitation of male sexual behavior in mice Journal of Comparative and Physiological Psychology 93 211-222

deCatanzaro D and MacNiven E (1992) Psychogenic pregnancy disruptions in mammals Neuroscience and Biobehavioral Reviews 16 43-53

deCatanzaro D and Storey AE (1989) Partial mediation of strange-male-induced pregnancy blocks by sexual activity in mice Journal of Comparative Psychology 103 381-388

deCatanzaro D, MacNiven E and Ricciuti F (1991) Comparison of the adverse effects of adrenal and ovarian steroids on early pregnancy in mice Psychoneuroendocrinology 16 525-536

deCatanzaro D, MacNiven E, Goodison T and Richardson D (1994) Estrogen antibodies reduce vulnerability to stress-induced failure for intrauterine implantation in inseminated mice Physiology and Behavior 55 35-38

deCatanzaro D, Muir C, O'Brien J and Williams S (1995a) Strange-male-induced pregnancy disruption in mice: reduction of vulrerability by $17 \beta$-estradiol antibodies Physiology and Behavior 58 401-404

deCatanzaro D, Wyngaarden P, Griffiths J, Ham M, Hancox J and Brain D (1995b) Interactions of contact, odor cues, and androgens in strange-male-induced early pregnancy disruptions in mice (Mus musculus) Journal of Comparative Psychology 109 115-122

Dominic CJ (1965) The origin of pheromones causing pregnancy block in mice Journal of Reproduction and Fertility $10469-472$

Dominic CJ (1966a) Observations on the reproductive hormones of mice Journal of Reproduction and Fertility 11 407-414

Dominic CJ (1966b) Observations on the reproductive pheromones of mice II Journal of Reproduction and Fertility 11 415-421 
Drickamer LC (1989) Pregnancy block in wild stock house mice, Mus domesticus: olfactory preferences of females during gestation Animal Behaviour 37 690-692

Fekete E (1941) Histology. In Biology of the Laboratory Mouse pp 89-167 Ed. GD Snell. Dover Publications, New York

Gawienowski AM, Orsulak M, Stacewicz-Sapuntzakis M and Joseph BM (1975) Presence of sex pheromones in preputial glands of male rats Journal of Endocrinology $67283-288$

Harper MJK (1967) Effects of androstenedione on pre-implantation stages of pregnancy in rats Endocrinology 81 1091-1098

Hsu C-Y (1948) Influence of temperature on development of rat embryos Anatomical Record 100 79-90

Hucklebridge FH, Nowell NW and Wouters A (1972) A relationship between social experience and preputial gland function in the albino mouse Journal of Endocrinology 55 449-450

Jones RB and Nowell NW (1973) Effects of preputial and coagulating gland secretions upon aggressive behaviour in male mice: a confirmation Journal of Endocrinology 59 203-204

Keverne EB and de la Riva C (1982) Pheromones in mice: reciprocal interactions between nose and brain Nature 296 148-150

Lloyd-Thomas A and Keverne EB (1982) Role of the brain and accessory olfactory system in the block to pregnancy in mice Neuroscience 7 907-913

McGill TE (1965) Studies of the sexual behavior of male laboratory mice: effect of genotype, recovery of sex drive, and theory. In Sex and Behavior pp 76-88 Ed. FA Beach. Wiley, New York

McKinney TD and Christian JJ (1970) Effect of preputialectomy on fighting behavior in mice Proceedings of the Society of Experimental Biology and Medicine 134 291-293

Marchlewska-Koj A (1977) Pregnancy block elicited by urinary proteins of male mice Biology of Reproduction 18 729-732

Marchlewska-Koj A (1981) Pregnancy block elicited by male urinary peptides in mice Journal of Reproduction and Fertility $61221-224$

Marchlewska-Koj A, Pochron E and Sliwowska A (1990) Salivary glands and preputial glands of males as source of estrus-stimulating pheromone in female mice Journal of Chemical Ecology 16 2817-2822

Moore CL and Samonte BR (1986) Preputial glands of infant rats provide chemosignals for maternal discrimination of sex Journal of Comparative Psychology 100 76-80
Mugford RA and Nowell NW (1970) Endocrine control over production and activity of the anti-aggression pheromone from female mice Journal of Endocrinology $49225-232$

Mugford RA and Nowell NW (1971) The preputial glands as a source of aggression-promoting odors in mice Physiology and Behavior 6 247-249

Noble RL and Collip JB (1941) A possible direct control of the preputial glands of the female rat by the pituitary gland and indirect effects produced through adrenals and gonads by augmented pituitary extracts Endocrinology 29943

Orsulak PJ and Gawienowski AJM (1972) Olfactory preferences for the rat preputial gland Biology of Reproduction 6 219-223

Parkes AS and Bruce HM (1962) Pregnancy-block in female mice placed in boxes soiled by males Journal of Reproduction and Fertility 4 303-308

Rajendren G and Dominic CJ (1986) Effect of bilateral transection of the lateral olfactory tract on the male-induced implantation failure (the Bruce Effect) in mice Physiology and Behavior 36 587-590

Raso E, Timar J and Lapis K (1992) Development and characterization of a sex-dependent metastatic preputial gland adenocarcinoma in human tumorbearing immunosuppressed F344 rats Carcinogenesis 13 1281-1284

Rosser AE, Remfry CJ and Keverne EB (1989) Restricted exposure of mice to primer pheromones coincident with prolactin surges blocks pregnancy by changing hypothalamic dopamine release Journal of Reproduction and Ferility 87 $553-559$

Runner ML (1959) Embryocidal effects of handling pregnant mice and its prevention with progesterone Anatomical Record 133 330-331

Thody AJ and Dijkstra H (1978) Effect of ovarian steroids on preputial gland odours in the female rat Journal of Endocrinology 77 397-403

Vandenbergh JG (1967) Effect of the presence of a male on the social maturation of female mice Endocrinology 81 345-359

Vandenbergh JG (1969) Male odor accelerates female sexual maturation in mice Endocrinology $84658-660$

Whitten WK (1956) Modification of the oestrous cycle of the mouse by external stimuli associated with the male Journal of Endocrinology 13 399-404

Wiebold JL, Stanfield PH, Becker WC and Hillers JK (1986) The effect of restraint stress in early pregnancy in mice Journal of Reproduction and Fertility $\mathbf{7 8}$ 185-192

Zondek B and Tamari I (1967) Effects of auditory stimulation on reproduction Ciba Foundation Study Group 26 4-19 